Les défis que le système de santé suisse devra relever ces prochaines années appellent des solutions auxquelles tous les partenaires clés puissent apporter leur contribution. Le conseiller fédéral Pascal Couchepin ne semble malheureusement pas être de cet avis. II décide sans consulter les partenaires impliqués - et sans envisager ses décisions dans un contexte global comme il le faudrait. La révision de la Liste des analyses n'est qu'un exemple parmi d'autres, mais pour le corps médical c'est un exemple de trop. La FMH estime que le temps est venu d'un changement de culture au sein du Département fédéral de l'intérieur. A la demande de l'Assemblée des délégués, nous avons exprimé cette exigence avec la fermeté nécessaire dans un communiqué de presse daté du 22 février. Nous souhaitons donner l'occasion à tous les lecteurs et lectrices du BMS que le sujet intéresse de lire ce texte important.

Dr Jacques de Haller, président de la FMH

\title{
L'incompétence règne dans le département de Pascal Couchepin
}

La Fédération des médecins suisses (FMH) est inquiète: le conseiller fédéral Pascal Couchepin et l'Office fédéral de la santé (OFSP) qui lui est subordonné prennent de plus en plus souvent des décisions arbitraires révélatrices d'incompétence. Ils ne tiennent pas compte des expertises ou recommandations des partenaires impliqués et les rejettent de façon autoritaire. Ce faisant, ils portent préjudice au système de santé suisse. La FMH estime dès lors que la position du conseiller fédéral Pascal Couchepin n'est plus acceptable. Pour que la population suisse puisse continuer à compter sur des soins de santé performants et de qualité dans tout le pays, il est impératif que le Département fédéral de l'intérieur adopte une nouvelle culture de communication.

Département Communication

Correspondance:

$\mathrm{FMH}$

Communication

Elfenstr. 18

3000 Berne 15

kommunikation@fmh.ch
C'est à fin janvier que le ministre de la santé a décidé de réduire de $20 \%$ en moyenne les tarifs des analyses de laboratoire en Suisse. Le fait qu'environ 7500 laboratoires de cabinet médical ne pourront désormais plus couvrir leurs coûts et devront simplement fermer, à terme, semble lui être complètement indifférent. Tout comme il a ignoré la recommandation des experts de la Commission fédérale des analyses, moyens et appareils (CFAMA) chargée de le conseiller, Pascal Couchepin n'a pas tenu compte non plus du modèle de remplacement mis au point par le corps médical. Or ce modèle de coûts, intitulé «Tarif Point-ofCare», proposait des tarifs calculés selon des critères d'économie d'entreprise qui auraient été corrects pour toutes les parties impliquées. «L'Etat c'est moi, je décide seul!» Et le résultat de cette attitude ressort de l'examen détaillé du modèle proposé par l'OFSP: la FMH y a vérifié neuf des analyses les plus courantes dans les soins de basesept d'entre elles sont clairement entachées d'erreurs. Un simple exemple: le nouveau tarif prévoit une centrifugation dans le cadre du test servant à déterminer le taux sanguin de glycémie (HbA1c), alors que ce procédé rend l'échantillon inutilisable!

Et ce n'est pas tout: le nouveau tarif de laboratoire débouche sur d'incroyables résultats paradoxaux: alors que deux tiers des médecins enregistrent une baisse du chiffre d'affaires de leur laboratoire de 15 à $35 \%$, un tiers d'entre eux le voient augmenter jusqu'à $40 \%$ par rapport à la situation actuelle! C'est ce que révèle une étude fondée sur la base des données de la Caisse des médecins de l'année 2007, et qui a calculé le chiffre d'affaires de quelque 2400 laboratoires de cabinet selon le nouveau tarif. Au lieu de solliciter la collaboration des partenaires impliqués, le 
conseiller fédéral Pascal Couchepin et l'OFSP ont gardé secrètes leurs bases de calcul jusqu'au dernier moment. Ce sont malheureusement les patients qui en feront les frais.

Le conseiller fédéral Couchepin semble avoir perdu la maîtrise d'autres dossiers encore. Il a annoncé fin janvier vouloir soutenir les médecins généralistes par une augmentation des indemnités pour urgence à partir du $1^{\mathrm{er}}$ mars 2009. Or il ne fait que remettre ce tarif à son niveau d'origine - ainsi qu'en avaient convenu les partenaires tarifaires compétents - après qu'une baisse introduite en 2007 avait causé des pertes massives aux médecins. On corrige aussi sans complexe dans le domaine de la psychothérapie médicale: l'OFSP s'affaire actuellement à annuler les articles introduits au $1^{\text {er }}$ janvier 2007 dans l'Ordonnance sur les prestations de l'assurance des soins (OPAS), qui obligent les psychiatres à demander l'accord d'un médecin-conseil lorsqu'une thérapie requiert plus de dix séances. Le corps médical avait fait remarquer très tôt que cette règle n'avait pas de sens. Une règlementation semblable dans le domaine de la physiothérapie et de l'ergothérapie s'avère tout aussi inutilisable. Dans ce cas aussi, l'OFSP a mis en route une procédure de correction et a tout récemment donné l'ordre de ne plus appliquer les articles correspondants de l'OPAS.

Les décisions arbitraires prises en politique tarifaire par le conseiller fédéral Couchepin illustrent de façon saisissante à quel point son action est irréaliste. Dans le domaine de la santé, son département fait figure de chantier sans fin: aucune solution convaincante n'a encore été trouvée pour remplacer la clause du besoin frappant les médecins qui arrivera à échéance à fin 2009. Et aucune réglementation constructive n'a vu le jour non plus en matière de compensation des risques. Le ministre de la santé a oublié que c'est la population suisse qui devrait être au centre de la politique de la santé de notre pays. En solitaire, il s'acharne sur tel ou tel tarif avec l'OFSP et en perd la vue d'ensemble. C'est pourquoi la FMH lui suggère de céder la place à des forces vives - pour que l'esprit d'ouverture et de dialogue retrouve sa place au Département fédéral de l'intérieur. 